\title{
The Use of 4G Android Tablets for Enhanced Patient Activation of Chronic Disease Self-Management in People with Heart Failure
}

Judith Kutzleb*,Nancy Elmann, Andrew Fruhschien, Stephen Angeli, Angel Mulkay , Jarrett Bauer, Rohan Udeshi and Dan Priece

Advanced Practice Professionals at Holy Name Medical Center, 718 Teaneck Road, Teaneck, New Jersey, USA

"Corresponding author: Judith Kutzleb, et al. Advanced Practice Professionals at Holy Name Medical Center, 718 Teaneck Road, Teaneck, New Jersey, 07666, USA, Tel: (973) 600-5991; E-mail: kutzleb@mail.holyname.org

Received date: March 10, 2014; Accepted date: April 28, 2014; Published date: May 03, 2014

Copyright: (C) 2014 Kutzleb J, et al. This is an open-access article distributed under the terms of the Creative Commons Attribution License, which permits unrestricted use, distribution, and reproduction in any medium, provided the original author and source are credited.

\begin{abstract}
Problem

The purpose of this research was to evaluate the impact of an advanced practice nurse-directed patient education approach to heart failure treatment integrating the use of an interactive $4 \mathrm{G}$ android tablet, will enable patients to experience enhanced patient activation and engagement in chronic disease self-management and fewer 30-day rereadmissions.
\end{abstract}

\section{Data source}

This was a prospective patient randomization, multi-center quasi-experimental design study of 50 patients comparing an advanced practice nurse-directed education of disease self-management and use of a $4 \mathrm{G}$ android tablet $(\mathrm{TC})$ group $(\mathrm{n}=25)$ and routine medical management $(\mathrm{MC})$ group. The study length was 12 months.

\section{Conclusions}

Descriptive statistics were computed, and the intervention and control groups were compared for differences. Descriptive statistics using ANOVA was conducted to calculate for statistical significance of readmissions between the two groups at 30 days. T-tests showed that the 30 -day readmissions rate was significantly lower for the tablet groups compared to the medical group at 30 days ( $8 \%$ and $28 \%$ respectively; $\mathrm{P}=0.010$ ).

\section{Implications for Practice}

The results support that integrating $4 \mathrm{G}$ android tablet technology does have a significant impact on enhancing patient activation and engagement in chronic disease self-management and correlated to reduced 30-day readmissions in people with heart failure.

Keywords Heart failure; Patient education; Mobile technology in chronic disease; Patient activation; Patient engagement; Disease selfmanagement

\section{Introduction}

Heart Failure (HF) is a major public health problem in the United States and is the most common discharge diagnosis in the population of people over 65 years. The prevalence of heart failure continues to rise involving approximately 6.6 million Americans, with 550,000 newly diagnosed cases each year $[1,2]$. Because of the aging population and despite the successful treatment of underlying conditions that cause heart failure, such as coronary artery disease and hypertension, the number of people with heart failure continues to increase. The annual number of hospitalizations for the primary diagnosis of heart failure has increased from 800,000 to over 1 million over the last 20 years [3]. Economically, heart failure is the most costly healthcare problem, with direct cost for diagnosis and treatment being approximately $\$ 39.2$ billion in 2012 [1]. Of particular concern to healthcare organizations, are the frequent readmissions despite the advancements in HF-related medical care [2]. Approximately one fifth of Medicare beneficiaries discharged from a hospital are readmitted within 30 days; and $50 \%$ of patients with HF are readmitted within 6 months of discharge, with $70 \%$ of the rehospitalizations being related to worsening HF symptomatology [4].

Many healthcare organizations are focusing on improving performance and patient outcomes in HF paying particular attention to better chronic disease management so as to prevent readmissions, decrease costs, and improve quality of life [5-7]. As of October 2013, the Centers for Medicare and Medicaid Services (CMS) have implemented decreased reimbursement with additional penalties for patients who require readmission for any cause within 30 days of their initial admission [8]. A decrease in healthcare reimbursement can be a threat to most hospitals' mission of patient care. Financial pressures may stem from reduced insurance reimbursement, capitation, and changes in public funding. Pressures for innovation result from increasing numbers of patients, higher acuity, and the aging population with complex chronic disease processes [9-11]. 
Page 2 of 5

Primary care providers, not cardiologists, treat most patients and play a key role in improving clinical outcomes in patients with and at risk for heart failure. Educating patients about their disease; motivating adherence to a course of therapy through patient activation of disease self-management are critical aspects in promoting positive outcomes. The purpose of this project was two-fold: first, to evaluate the impact of nurse-directed patient education on chronic disease selfmanagement; second, to evaluate the extent to which 30 day hospital readmissions decreased by patient activation in disease selfmanagement through the use of a $4 \mathrm{G}$ android tablet. Patient activation is defined as, an individual being able to self-manage symptoms and problems; engage in activities that maintain physical function; and maintain involvement in clinical decision-making as their overall reduced health declines $[11,12]$.

\section{Literature Review}

Mobile technology has expanded dramatically around the world. The integration of $3 \mathrm{G}, 4 \mathrm{G}$, and bluetooth technology has had a huge impact on chronic disease management $[13,14]$. Technology has been used to support lifestyle modification for diabetes and COPD disease management $[15,16]$. Researchers have developed mobile phone software applications that can help COPD patients self-manage their disease [17-19]. The aim is to provide software applications that are available any time and any place to help individuals improve their health status and obtain social support while at the same time providing ready access to a health professional if an emergency arises $[20,21]$. One of the biggest problems for elderly patients is forgetting to take their prescription medications. It is estimated that only 50 percent of patients take their medication as prescribed [22,23]. They either forgot to take their medication or did not take them at the time or dosage set by their primary care provider. This means that half of the benefit of prescription medications is lost through human error. Research has found that individuals who have access and used technology that allows for self-management of disease, have been able to obtain some level of success in terms of improving their health outcomes [24,25].

Liu et al., conducted a study on mobile phones plus COPD-specific software. The results of this study did show improvement with incremental shuttle walk test distances, walking endurance, compliance with home-based exercise programs, inspiratory capacity, quality of life scores, exercise time, physical functioning and selfefficacy in managing dyspnea. The results also showed decreased hospital length of stays for disease exacerbations as well as the number of unscheduled visits to physician offices.

Cotter et al. 2013, did a systematic review of evidence on internet interventions to support lifestyle modification for diabetes management. The results of the systematic review demonstrated improvements in diet and or physical activity, and improvements in glycemic control when comparing web-based intervention with control cohorts. Therefore, in conclusion, web-based strategies provided a valuable option for facilitating diabetes self-management.

Johnston et al., examined whether the use of a Blackberry-based daily symptom diary would detect $95 \%$ or more COPD exacerbations and enable characterization of seasonal differences among daily diary entries. An alert system was triggered when symptoms changed, missed diary transmissions occurred, or there was need for medical intervention. The results showed that of the 28,514 possible daily symptoms diary entries, the participants transmitted $99.9 \%$ of the possible entries. In conclusion, smart-phone based collection of COPD symptom diaries enabled near-complete identification of exacerbations at inception.

\section{Treatment Approaches}

Pharmacotherapeutic treatments for heart failure have improved outcomes in the past 10 years, and evidence-based guidelines provide a structured approach to the treatment of heart failure. Clinical trials have shown that maximizing pharmacotherapy in conjunction with patient education and activation reduces hospital admissions, decreases morbidity and mortality, and improves the quality of life for patients with heart failure. An advanced practice nurse-directed multidisciplinary approach to heart failure management incorporates both patient monitoring and patient self-management of symptoms as a central strategy. Active patient activation is paramount in the effective management of chronic conditions. Various selfmanagement initiatives have been developed to assist patients to optimize the management of their health. The objective of these programs is to provide patients with information and skills that will enhance their ability to participate in their health care. Targeting risk factors for unplanned readmissions in concert with protocol driven medical management are the tenets for achieving successful outcomes.

\section{Hypothesis}

Patients who participate in an advanced practice nurse-directed patient education approach to heart failure treatment in concert with the use of an interactive $4 \mathrm{G}$ android tablet will experience enhanced patient activation and engagement in disease self-management and fewer 30 day hospital readmissions.

\section{Research Methods}

This study is a prospective multi-center quasi-experimental design with patient randomization to an advanced practice nurse-directed disease self-management with the use of a $4 \mathrm{G}$ Android Tablet (TC) group and a routine medical management (MC) group.

\section{Sample}

All adult patients 20 to 89 years of age admitted to the hospital with the principal diagnosis of heart failure were considered for inclusion in the study. The design flow chart (Figure 1) was developed to identify the process of patients through the study. Patient referrals were from either cardiologists or primary care physicians. Both sites were comparable in the medical management and patient population mix of heart failure admissions.

All patients enrolled had a diagnosis of heart failure confirmed by a cardiologist based on echocardiography with evidence of left ventricular systolic dysfunction or diastolic dysfunction. Patients who were not literate in English were excluded. Patients presenting with heart failure in the setting of myocardial infarction or unstable angina or in whom failure was not thought to be the primary problem, or for whom heart failure was a secondary diagnosis in conjunction with multiple chronic comorbidities, were excluded. Also, patients with illnesses that could compromise survival over the duration of the study (e.g. cancer) or with cognitive impairment, or who were taking moodaltering medications (antipsychotics, antianxiety, and antidepressant agents) were excluded. The baseline characteristics gathered on the total population, TC group and MC group included gender, age, 
Page 3 of 5

average ejection fraction percentage, New York Heart Association (NYHA) classification, etiology, risk factors, and medications (Table 1).

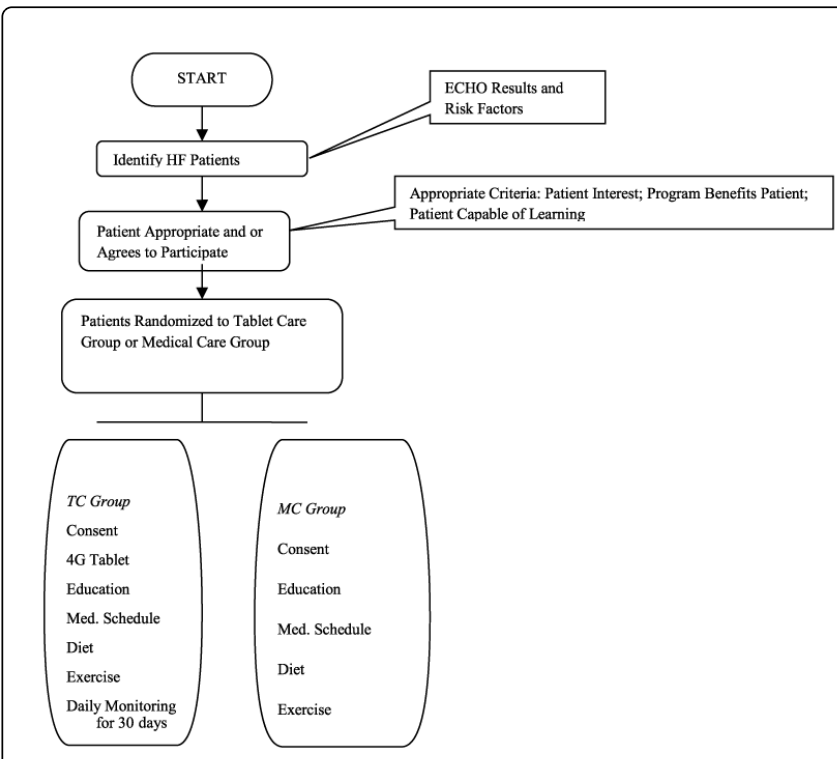

Figure 1: Program Design Flow Chart.

\begin{tabular}{|l|l|l|l|l|}
\hline Demographics & TC group & $\%$ & MC group & $\%$ \\
\hline Gender & & & & \\
\hline Male & 14 & $28 \%$ & 13 & $26 \%$ \\
\hline Female & 11 & $22 \%$ & 12 & $24 \%$ \\
\hline Average Age & 71 & & 72 & \\
\hline Average EF (\%) & $37 \%$ & & $41 \%$ & \\
\hline NYHA class & & & & \\
\hline Class I & 0 & & 0 & \\
\hline Class II & 12 & $24 \%$ & 15 & $30 \%$ \\
\hline Class III & 9 & $18 \%$ & 7 & $14 \%$ \\
\hline Class IV & 4 & $8 \%$ & 3 & $6 \%$ \\
\hline Etiology of HF & & & & \\
\hline Ischemic CM & 4 & $8 \%$ & 1 & $2 \%$ \\
\hline Hypertension & 10 & $20 \%$ & 15 & $30 \%$ \\
\hline Alcoholic CM & 3 & $6 \%$ & 0 & $0 \%$ \\
\hline Idiopathic & 5 & $10 \%$ & 3 & $6 \%$ \\
\hline Valvular & 2 & $4 \%$ & 4 & $8 \%$ \\
\hline Risk Factors & & & 14 & $28 \%$ \\
\hline Hypertension & 21 & $42 \%$ & 11 & $22 \%$ \\
\hline DM & 11 & $22 \%$ & 11 & \\
\hline Smoking & 8 & $16 \%$ & & \\
\hline
\end{tabular}

\begin{tabular}{|l|l|l|l|l|}
\hline Alcohol abuse & 1 & $2 \%$ & 3 & $6 \%$ \\
\hline CAD & 16 & $32 \%$ & 12 & $24 \%$ \\
\hline COPD & 7 & $14 \%$ & 5 & $10 \%$ \\
\hline Renal Insufficiency & 4 & $8 \%$ & 3 & $6 \%$ \\
\hline Medications & & & & \\
\hline ACE inhibitor & 23 & $46 \%$ & 24 & $48 \%$ \\
\hline Diuretic & 25 & $50 \%$ & 23 & $46 \%$ \\
\hline Beta-blocker & 25 & $50 \%$ & 25 & $50 \%$ \\
\hline Digoxin & 5 & $10 \%$ & 7 & $14 \%$ \\
\hline Other & 10 & $20 \%$ & 8 & $16 \%$ \\
\hline
\end{tabular}

Table 1: Baseline demographic characteristics of total population, TC group, and MC group

Note: N=50; CM: Cardiomyopathy; NYHA: New York Heart Association; EF: Ejection Fraction; ACE: Angiotensin-Converting Enzyme; DM: Diabetes Mellitus; CAD: Coronary Artery Disease; COPD: Chronic Obstructive Pulmonary Disease

After blind randomization, all patients who participated were told the purpose of the study and signed an informed voluntary written consent form (approved by the institutional review boards) indicating their willingness to participate. Patients were informed that their responses would be kept confidential and that under no circumstances would their participation or lack of participation influence treatment decisions or jeopardize their care. Patients were interviewed to determine their hospitalization history and the length and nature of symptoms associated with heart failure. Severity of symptoms was assessed by categorizing the patients according to the NYHA functional classifications: class I, unlimited activity without cardiac symptoms; class II, ordinary physical activity causes fatigue, dyspnea, or palpitations; class III, marked limitation of activity and symptoms with less than usual activity but no symptoms at rest; and class IV, any physical activity is accompanied by symptoms and symptoms may occur at rest.

\section{Setting and Plan of Action}

The patients were evaluated by the advanced practice nurse after admission in each study site. At the time of evaluation, patients randomized to the TC group were informed of the following for study inclusion: education on the use of the $4 \mathrm{G}$ android tablet for recording of daily weights; daily exercise; and daily medication administration for the duration of 30 days. The TC group also received comprehensive disease education with a patient education pamphlet describing heart failure, listing recommendations to maintain a heart healthy lifestyle and signs and symptoms of changes in condition status that warrants medical notification. Medication compliance counseling consisted of a personalized medication schedule. Each individual schedule was programmed into the tablet where the patient was required to record his/her administration of medications within the established parameters. The tablet also contained pre-recorded videos and power points for educational support for both the patient and family members reinforcing the importance of medication adherence; daily weight monitoring; meal planning and managing a low-sodium diet. Predischarge counseling also concentrated on 
Page 4 of 5

smoking cessation and elimination of alcohol intake. Interventions were monitored by the advanced practice nurse on a daily basis by review of the computer generated program of the data obtained from the android tablets.

The MC group was informed of the following criteria: review of the patient education booklet that included daily weight charting; diet and nutrition counseling incorporating food exchange list, food preparation tips, and a four-step approach to managing a low-salt diet. A medication administration schedule was designed for each patient, listing medications, dosage strengths, and mechanism of action. Individualized counseling concentrated on exercise, smoking cessation, and elimination of alcohol intake. Interventions were monitored by telephone follow-up on a weekly basis by the call center from each site. A standard questionnaire was utilized to maintain consistency in patient response.

\section{Functional Capacity}

The objective measure of functional capacity was obtained using a 6-minute walk test. Patients were told to walk at a brisk pace as far as they could within the allotted time, resting as needed. The limitation of the 6-minute walk test is the inability to standardize it with regard to step length and pace. However, differences were minimized in the method at each site by using the same physical area each time, and by standardizing the directions given to the patients.

\section{Results}

\section{Characteristics of Sample}

Out of 83 eligible patients, 50 patients ( 25 from site \#1 and 25 from site \#2) consented for study participation. The mean age of the study participants was 71 years old; $46 \%$ were women and $54 \%$ were men. Average ejection fraction for participants in the TC group was $37 \%$ versus $41 \%$ in the MC group, which represents a significantly higher risk population in the TC group. There was no significant difference found by group membership regarding gender or race. The average number of medications taken was six and is consistent with the complex treatment that is characteristic of this illness. In terms of illness management, $80 \%$ of the MC either reported never being placed on or did not follow a strict low-sodium diet, and compliance to medication adherence was low. Seventy-seven percent of the TC groups and $20 \%$ of the MC group engaged in daily exercise activity. Eighty percent of the MC group managed their illness by restricting their activities and taking frequent rests. There were no deaths, nor any patients lost to follow-up in either group during this study. Age, race, and economic status did not differ significantly between patients who participated in the study.

The data was analyzed using the Statistical Package for Social Sciences 21 (IBM Corp.) for analysis. Descriptive statistics were computed, and the intervention and control groups were compared for differences. Descriptive statistics using ANOVA was conducted to calculate for statistical significance of readmissions between the two groups at 30 days. T-tests showed that the 30 -day readmissions rate was significantly lower for the tablet group compared to the medical group at 30 days ( $8 \%$ and $28 \%$ respectively; $\mathrm{P}=0.010$ ) (see Table 2 ).

\begin{tabular}{|c|c|c|c|c|}
\hline $\begin{array}{l}\text { Variable } \\
\text { (Tablet Care\%/ } \\
\text { Medical Care \%) }\end{array}$ & $F$ & df & $95 \% \mathrm{Cl}$ & $\begin{array}{l}\text { Standard } \\
\text { Deviation }\end{array}$ \\
\hline
\end{tabular}

\begin{tabular}{|l|l|l|l|l|l|}
\hline 30 days $(8 \% / 28 \%)$ & 1.40 & 1 & $1.4209-1.6286$ & .40118 & .010 \\
\hline
\end{tabular}

Table 2: One-Way ANOVA Statistical Analysis of HF Readmissions between Tablet Care group and Medical Care group at 30 days

\section{Note: $\mathrm{F}=$ ratio; $\mathrm{df}=$ degrees of freedom. ${ }^{\star} \mathrm{P}<.05$ (two-tailed test)}

The results support that integrating $4 \mathrm{G}$ android tablet technology does have a significant impact on enhancing patient activation and engagement in chronic disease self-management and correlated to reduced 30-day readmissions in people with heart failure.

\section{Discussion}

The research interventions in this study were directed toward increasing patient activation and engagement with the use of technology as an adjuvant modality to patient education of chronic disease self-management. This study focused specifically on patients with heart failure who were identified to be at high-risk for early readmissions, and the intervention with technology addressed targeted causes of re-hospitalization (life-style, medication and diet noncompliance, exercise noncompliance, and lack of engagement for self-care disease management). The study sites were initiated in hospitalized heart failure patients who were discharged home, whereas prior studies focused on interventions on hospitalized heart failure patients not including post-acute discharge management or monitoring. The literature has theorized that outpatient management should be successfully provided by primary care providers, not cardiologists. This study did prove that successful management of heart failure patients was provided by advanced practice nurses in concert with primary care providers. The outcome of this study also demonstrated that patient engagement did correlate with reduced 30day readmissions.

The TC group patients described their most positive changes in their overall well-being as relating to their ability to self-manage their medications and diet which stemmed from the daily engagement with the $4 \mathrm{G}$ interactive android tablet. The physiological changes experienced as a result of pharmacological and non-pharmacological regimens improved their overall exercise tolerance and ability to maintain independence. The improved adherence to treatment modalities as observed in this population has proven to be a predictor of adjustment to illness and disease self-management. The mean exercise time in the TC group increased from 6 minutes to 23 minutes daily. The 6-minute walk test proved to be an inexpensive and uncomplicated but limited clinical measure of exercise capacity.

\section{Future Research}

Further evaluation of functional capacity and NYHA classification to test for sensitivity for differences in clinical status of heart failure patients would require further study. Studies directed at integrating $4 \mathrm{G}$ android technologies for monitoring multiple disease processes in concert with HF management are required to identify if timely treatment of multiple disease exacerbations are associated with decreased risk for mortality and heart failure related hospitalizations. A number of patients could not be included in this study because of either language barrier or lack of health literacy. This represents a significant limitation of the present study given the high rate of functional illiteracy in the United States, along with the complexity of the illness management regimen needed for most heart failure patients. It is imperative that the needs of these patients be described 
and interventions for technology integration for chronic disease be developed for future studies. Last, further development of reliable and sensitive self-report and observer-related specific measures for study population would also be useful in the future.

\section{Conclusion}

In our opinion, the improved patient activation in our patients was a direct result of the study intervention with the use of $4 \mathrm{G}$ interactive android tablet. Patients were never without questions, and several patients were averted from either emergency room visits or rehospitalizations by timely interactions with the study team. The focused nature of the intervention, the close monitoring of patient activation through a web portal, and follow-up, support our hypothesis and explanations for our findings. A significant portion of patient crises are avoided through interventions that improve patient activation and compliance with prescribed management protocols and improve recognition of early warning signs of clinical problems.

Thus, as management of symptoms and the work associated with the regimen needed to achieve symptom control are necessary elements in successful management of any chronic illness, heart failure patients participating in an advanced practice nurse symptom management program in concert with interactive technology can be expected to achieve better outcomes than patients who do not learn to actively engage in disease self-management. The participants in this study were able to demonstrate disease self-management through daily entry of prescribed activities of daily weights, medication regimens (dosages and administration), low sodium diet adherence, and lifestyle modification via the use of $4 \mathrm{G}$ android tablets. The study demonstrated the use of interactive technology significantly improved patient engagement, and prevented 30-day rehospitalizations in patients with heart failure.

\section{Conflict of Interest Statement}

No relationship that might represent a conflict of interest exists between the authors and any commercial entity or product mentioned in this manuscript. No commercial entity has made any inducements to submit this article for publication.

\section{References}

1. Roger VL, Go AS, Loyd-Jones DM, Benjamin EJ, Berry JD (2012) Heart disease and stroke statistics: 2012 update: a report from the American Heart Association. Circulation 125: e2-e220.

2. Butler J, Kalogeropoulos A (2008) Worsening heart failure hospitalization epidemic we do not know how to prevent and we do not know how to treat! J Am Coll Cardiol 52: 435-437.

3. Ross JS, Chen J, Lin Z, Bueno H, Curtis JP, et al. (2010) Recent national trends in readmission rates after heart failure hospitalization. Circ Heart Fail 3: 97-103.

4. Jencks SF, Williams MV, Coleman EA (2009) Rehospitalizations among patients in the Medicare fee-for-service program. N Engl J Med 360: 1418-1428.

5. Kutzleb J, Reiner D (2006) The impact of nurse-directed patient education on quality of life and functional capacity in people with heart failure. J Am Acad Nurse Pract 18: 116-123.

6. Fang J, Mensah GA, Croft JB, Keenan NL (2008) Heart failure-related hospitalization in the U.S., 1979 to 2004. J Am Coll Cardiol 52: 428-434.

7. Dickson VV, Riegel B (2009) Are we teaching what patients need to know? Building skills in heart failure self-care. Heart Lung 38: 253-261.
8. Medicare Payment Advisory Commission (2008) Report to Congress: Reforming the delivery system.

9. Kim, S, Han H (2013). Evidence-based strategies to reduce readmissions in patients with heart failure. The Journal for Nurse Practitioners, 9: 224-232.

10. Jessup M, Abraham W, Casey D, Arthur M. Feldman, Theodore G Ganiats, (2009) Focused Update: ACCF/AHA guidelines for the diagnosis and management of heart failure in adults: A report of the American College of Cardiology Foundation/American Heart Association Task Force on Practice Guidelines: Developed in collaboration with the international society of Heart and lung transplantation. Circulation 119: 1977-2016.

11. Hibbard JH, Stockard J, Mahoney ER, Tusler M (2004) Development of the patient activation measure (PAM): Conceptualizing and measuring activation in patients and consumers. Health Services Research 39: 1005-1026.

12. Gerber LM, Barrón Y, Mongoven J, McDonald M, Henriquez E, et al. (2011) Activation among chronically ill older adults with complex medical needs: challenges to supporting effective self-management. J Ambul Care Manage 34: 292-303.

13. Noh JH, Cho YJ, Nam HW, Kim JH, Kim DJ, et al. (2010) Web-based comprehensive information system for self-management of diabetes mellitus. Diabetes Technol Ther 12: 333-337.

14. Yu CH, Bahniwal R, Laupacis A, Leung E, Orr MS (2012) Systematic review and evaluation of web-accesible tools for management of diabetes and related cardiovascular risk factors by patients and healthcare providers. J Am Med Inform Assoc 19: 514-522.

15. Polisena J, Tran K, Cimon K, Hutton B, McGill S (2010) Home telehealth for chronic obstructive pulmonary disease: a systematic review and metaanalysis. J Telemed and Telecare, 16: 120-127

16. Trappenburg J, Niesink A, de Weert-van Oene G, vander Zeijden H, van Snippenburg R, (2008) Effects of telemonitoring in patients with chronic obstructive pulmonary disease. Telemedicine Journal e-Health 14: 138-146.

17. Nguyen HQ, Wolpin S, Chiang KC, Cuenco D, Carrieri-Kohlman V (2006) Exercise and symptom monitoring with a mobile device. AMIA Annu Symp Proc .

18. Wang H, Liu J (2009) Mobile phone based health care technology. Recent patents on Biomedical Engineering 2: 15-21.

19. Feil EG, Glasgow RE, Boles S, McKay HG (2000) Who participates in Internet-based self-management programs? A study among novice computer users in a primary care setting. Diabetes Educ 26: 806-811.

20. Sala E, Alegre L, Carrera M, Ibars M, Orriols FJ, et al. (2001) Supported discharge shortens hospital stay in patients hospitalized because of an exacerbation of COPD. Eur Respir J 17: 1138-1142.

21. Bischoff EW, Boer LM, Molema J, Akkermans R, van Weel C, et al. (2012) Validity of an automated telephonic system to assess COPD exacerbation rates. Eur Respir J 39: 1090-1096.

22. World Health Organization (2003) Adherence to long-term therapies: evidence for action 7-10

23. Kaufman $\mathrm{N}$ (2010) Internet and information technology use in treatment of diabetes. Int J Clin Pract Suppl : 41-46.

24. Glasgow RE, Kurz D, King D, Dickman JM, Faber AJ, et al. (2010) Outcomes of minimal and moderate support versions of an internetbased diabetes self-management support program. J Gen Intern Med 25: 1315-1322.

25. Welch G, Shayne R (2006) Interactive behavioral technologies and diabetes self-management support: recent research findings from clinical trials. Curr Diab Rep 6: 130-136.

26. Liu WT, Wang CH, Lin HC, Lin SM, Lee KY, et al. (2008) Efficacy of a cell phone-based exercise programme for COPD. Eur Respir J 32: 651-659. 